\title{
Surgical pulmonary embolectomy and catheter-based therapies for acute pulmonary embolism: A contemporary systematic review
}

\author{
Pranav Loyalka, MD, ${ }^{\mathrm{a}}$ Muhammad Z. Ansari, MBBS, ${ }^{\mathrm{b}, \mathrm{c}}$ Faisal H. Cheema, MD, ${ }^{\mathrm{d}}$ \\ Charles C. Miller III, PhD, ${ }^{\mathrm{b}}$ Sudarshan Rajagopal, $\mathrm{MD}, \mathrm{PhD},{ }^{\mathrm{e}}$ and Keshava Rajagopal, $\mathrm{MD}, \mathrm{PhD}{ }^{\mathrm{b}, \mathrm{c}}$
}

\section{ABSTRACT}

Objectives: Mortality in acute pulmonary embolism (PE) is believed to be principally due to the subgroup of PEs that are massive. Systemic thrombolysis is the therapeutic mainstay for acute massive PE, despite evidence suggesting limited survival benefits. Both catheter-based therapies (CBT) and surgical pulmonary embolectomy (SE) are well-accepted alternatives to treat acute PE. However, the comparative effectiveness of these approaches is difficult to study. We conducted a systematic review of CBT and SE for acute PE.

Methods: The PubMed database was queried for CBT- and SE-related publications between January 1998 and June 2017. A minimum of 10 patients undergoing intervention(s) was required for inclusion, and studies must not have excluded patients with massive PE. End points examined included hospital mortality, and additionally for CBT, procedural success rate.

Results: A total of 75 studies (41 of CBT, 34 of SE) were identified, with 1650 patients undergoing CBT and 1101 undergoing SE. Patients undergoing SE were more critically ill than those undergoing CBT (massive PE, 545 out of 975 [55.9\%] for SE vs 742 out of 1553 [47.8\%] for CBT). Cardiopulmonary resuscitation (CPR) was required in 217 out of 1015 patients undergoing SE (21.4\%) versus 38 out of 983 patients undergoing CBT $(4.0 \%)$. The hospital mortality of SE was $14.0 \%$, versus $5.6 \%$ for CBT, in the entire patient group. However, the hospital mortality of SE in patients with pre-SE CPR was $46.3 \%$, whereas it was $6.8 \%$ in those patients without pre-SE CPR. Although CPR was associated with an increased risk of mortality both for CBT and SE, it accounted for all of the mortality effect on SE (the adjusted odds ratio for CPR in a random effects model with treatment considered was $9.79(95 \%$ confidence interval, 4.98-19.17; $P<.0001)$. The adjusted odds ratio for mortality for SE relative to CBT was 1.36 (95\% confidence interval, $0.80-2.32 ; P=.84$ ). Moreover, CBT was associated with a procedural failure rate of $8.3 \%$.

Conclusions: Both CBT and SE were associated with satisfactory published outcomes. SE is associated with greater absolute postprocedure mortality than CBT, but has been undertaken in more critically ill populations. The markedly higher incidence of CPR in SE accounts for the differential mortality between the patients undergoing SE and those undergoing CBT. Decision making with respect to best therapy must take into account potential needs for periprocedure artificial mechanical right ventricle and lung support, institutional experience and outcomes, anticipated therapeutic efficacy and benefit, and approach-specific risks. (J Thorac Cardiovasc Surg 2018;156:2155-67)

\footnotetext{
From the ${ }^{\mathrm{a}}$ Division of Cardiology, Department of Internal Medicine, and Departments of ${ }^{b}$ Cardiovascular and Thoracic Surgery and ${ }^{c}$ Advanced Cardiopulmonary Therapies and Transplantation, University of Texas-Houston McGovern Medical School and Memorial Hermann-Texas Medical Center, Houston, Tex; ${ }^{\mathrm{d} D i v i s i o n}$ of Cardiothoracic Transplantation and Circulatory Support, Texas Heart Institute at Baylor College of Medicine, Houston, Tex; and ${ }^{\mathrm{e} D i v i s i o n}$ of Cardiovascular Medicine, Department of Medicine, Duke University School of Medicine and Medical Center, Durham, NC.

Received for publication July 5, 2017; revisions received May 7, 2018; accepted for publication May 10, 2018; available ahead of print July 10, 2018.
}

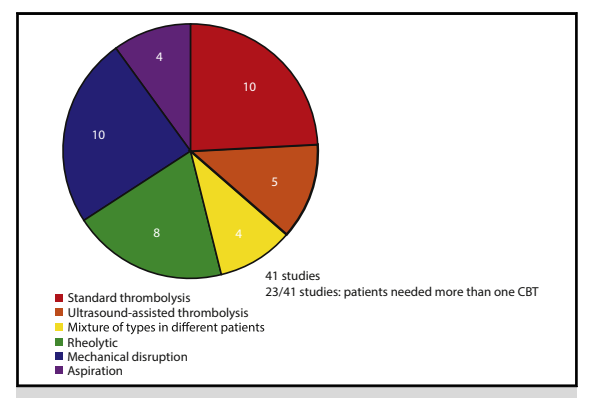

Heterogeneity of CBTs for acute PE.

\section{Central Message}

Outcomes of appropriately selected patients undergoing SE are at least equivalent to those undergoing CBTs to treat large-burden (ie, massive and submassive) PE.

\section{Perspective}

Both CBTs and SE have satisfactory published outcomes. Surgical therapy is associated with greater absolute postprocedure mortality than CBT, but has been undertaken in more critically ill populations. This accounts for the differential mortality of patients undergoing SE versus CBT.

See Editorial Commentary page 2168. 


$$
\begin{aligned}
& \text { Abbreviations and Acronyms } \\
& \text { CBT }=\text { catheter-based therapies } \\
& \text { CPR }=\text { cardiopulmonary resuscitation } \\
& \mathrm{ECMO}=\text { extracorporeal membrane oxygenation } \\
& \mathrm{PA} \quad=\text { pulmonary artery } \\
& \mathrm{PE} \quad=\text { pulmonary embolism } \\
& \mathrm{RV} \quad \text { right ventricle } \\
& \mathrm{SE} \quad=\text { surgical embolectomy }
\end{aligned}
$$

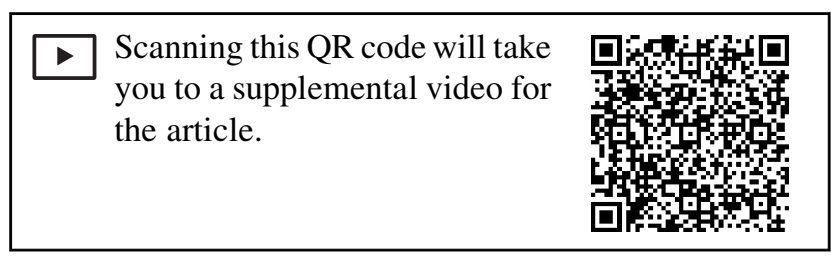

Acute pulmonary embolism (PE) continues to be among the leading causes of mortality and morbidity, despite state-of-the art pharmacologic and procedural therapies. In the United States, it is estimated that $\sim 400,000$ cases of acute PE are diagnosed, with an annual mortality of $\sim 50,000 .{ }^{1}$ The vast majority of mortality due to PE is believed to be due to massive PE, or PE that elevates right ventricular (RV) afterload sufficiently, such that major adverse hemodynamic sequelae (eg, low cardiac output syndrome manifesting with systemic arterial hypotension, and/or overt shock) occur. ${ }^{2-4}$ In addition, submassive $\mathrm{PE}$, or PE that elevates RV afterload such that evidence of $\mathrm{RV}$ dysfunction and/or injury is present, but in the absence of low-cardiac-output syndrome or shock (ie, in the absence of satisfying massive PE criteria), is also associated with an increased risk of mortality relative to uncomplicated PE. ${ }^{2-4}$ This is believed to be due to patients with submassive PE and high-risk features eventually deteriorating into massive PE physiology.

Standard-of-care therapy for patients with massive PE and submassive PE with high-risk features is centered on systemic thrombolysis, ${ }^{5,6}$ first implemented in the 1970s, and popularized in the late 1990s. However, thrombolytic agents do not improve survival in unselected patients with PE. ${ }^{7}$ Even in patients with massive and submassive $\mathrm{PE}$, although right-sided hemodynamic parameters and echocardiographic data are improved with thrombolysis, it is unclear whether thrombolytic agents actually improve overall survival. ${ }^{8,9}$ This is because although PE-related mortality may be improved by thrombolytic agents, this enhanced disease-specific mortality may be offset by intracranial hemorrhage-related mortality. ${ }^{8}$
In contrast to systemic fibrinolysis, the historical treatment for large-burden PE has been surgical embolectomy (SE). First attempted by Trendelenburg in 1908, successfully performed by Kirschner in 1924, and subsequently by Beall and Cooley using cardiopulmonary bypass, ${ }^{10} \mathrm{SE}$ has been associated with high mortalities in historical series and large contemporary databases. Kilic and colleagues ${ }^{11}$ reported $27 \%$ hospital mortality for SE in a study of the Nationwide Inpatient Sample, the largest series of SE examined to date. However, in centers with particular expertise in mechanical circulatory support/extracorporeal membrane oxygenation (ECMO), a recent resurgence in $\mathrm{SE}$ has been experienced; many studies (reviewed below) have demonstrated good results. Finally, in the background of historically suboptimal SE results, and deaths and complications arising from systemic administration of thrombolytic agents, a variety of minimally invasive catheter-based therapies (CBTs) have been developed. These include a wide range of techniques: catheterdirected infusion of thrombolytic agents, direct cathetermediated fragmentation, rheolytic catheter PE disruption, and suction embolectomy. ${ }^{12}$

To date, no comparative study of CBT has been conducted, and such a study would be difficult to conduct due to a sick patient population and significant variations in local practice. Still, with systemic fibrinolysis as the current standard, contemporary studies of CBT and SE are needed. We conducted a systematic review of the extant literature pertaining to CBT and SE in the era of systemic fibrinolysis.

\section{METHODS \\ Search Strategy}

An electronic search of the National Library of Medicine PubMed database was conducted (www.pubmed.gov). The beginning and ending time points for the search were January 1998 and June 2017. The beginning time point corresponds to being shortly after early stage and subsequent larger scale studies of systemic administration of thrombolytic agents in the treatment of acute PE. To identify CBT studies, the medical subject headings search terms used were pulmonary embolism OR pulmonary embolus AND embolectomy OR thrombectomy OR thrombolytic therapy AND catheter OR percutaneous OR mechanical. To identify SE studies, the medical subject headings search terms were pulmonary embolism OR pulmonary embolus AND embolectomy OR thromboembolectomy OR cardiopulmonary bypass. A total of 386 articles were initially identified in the CBT search strategy, and 511 were initially identified in the SE search strategy (by authors MZA, SR, and KR) (Figure 1). For CBT, studies were excluded if they were case reports or series $(<10$ patients $)(n=105)$, editorials or letters $(n=28)$, reviews $(n=83)$, not in English $(n=6)$, or did not clearly fit the inclusion criteria $(\mathrm{n}=123)$, with a final 41 studies for analysis (Figure 1, A). For SE, studies were excluded if they were case reports or series $(<10$ patients) $(n=136)$, editorials or letters $(n=26)$, reviews $(n=73)$, not in English $(n=60)$, or did not clearly fit the inclusion criteria $(\mathrm{n}=182)$, with a final 34 studies for analysis (Figure $1, B)$.

\section{Inclusion and Exclusion Criteria}

Articles were included if they were identified based on the initial search criteria, and if they satisfied the following inclusion criteria: either published in an English language journal or available in an English version, 

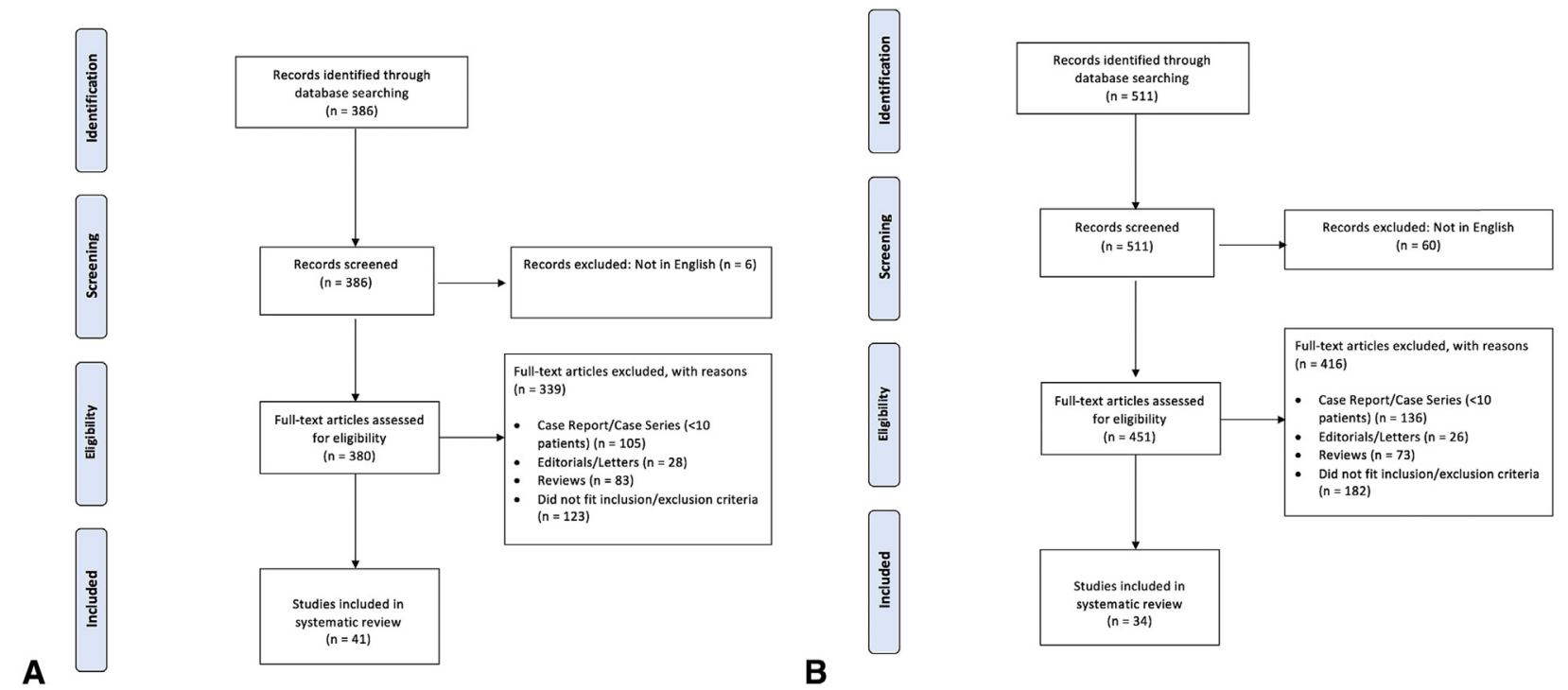

FIGURE 1. Preferred reporting items for systematic reviews and meta-analyses (PRISMA) flow diagrams for methods for treating pulmonary embolism. A, Catheter-based therapies. B, Surgical embolectomy.

contained $\geq 10$ patients who underwent procedural therapies, and did not exclude massive PE. Because false-positive results are possible; that is, SE articles could be identified within the CBT search and CBT articles could be identified within the SE search, studies were manually checked to determine whether in fact they belonged to the correct group (and were reassigned when appropriate). Finally, studies were manually checked to determine whether the same investigator groups and institutions published more than 1 study identified, with overlapping patients. If studies with overlapping patients were identified, only the most recent or largest study was chosen, excluding other studies. If overlapping patients were not identified; that is, totally chronologically sequential publications from an institution or investigator group, all pertinent publications were included.

\section{Massive and Submassive PE}

Massive and submassive PE were determined based on proposed definitions from a scientific statement from the American Heart Association published in 2011. ${ }^{13}$ Massive PE was characterized by evidence of low-cardiac-output syndrome or clinical shock attributed to $\mathrm{PE}$ as the underlying cause, based on 1 or more of the following: systemic arterial systolic blood pressure $<90 \mathrm{~mm} \mathrm{Hg}$, need for positive inotrope or systemic vasoconstrictor support, need for mechanical circulatory support, cardiac arrest, or profound bradycardia (heart rate $<40 \mathrm{bpm}$ ). This was with or without submassive PE criteria additionally being satisfied. Submassive PE was characterized by evidence of adverse effects on the $\mathrm{RV}$ (dysfunction and/or injury), in the absence of low-cardiac-output syndrome or clinical shock, based on 1 or more of the following: RV systolic hypokinesis, RV dilatation, elevated cardiac biomarkers (troponin I), elevated serum $\mathrm{N}$-terminal probrain natriuretic peptide, or electrocardiogram changes suggestive of RV strain. All publications were manually checked to determine possible discrepancies between the authors' interpretation of their data and the standardized definitions reviewed above.

\section{Statistical Analysis of the Effects of Cardiopulmonary Resuscitation on Outcomes}

Because none of the studies cited made head-to-head comparisons between CBT and SE, a traditional meta-analysis that summarizes within-study treatment effects is not possible. In addition, because the reports are all series, no control treatment common to both SE and CBT across publications (eg, systemic thrombolysis) could be identified for use in a network meta-analysis. We therefore combined the studies using simple crude proportion analyses, and conducted heterogeneity analyses using forest plots of proportions, with mixed-effect pooled estimates and $I^{2}$ measures. We further used mixed effects meta-regression models to estimate the adjusted marginal means as described by Madden and colleagues, ${ }^{14}$ and we followed the conceptual framework described by Benedetto and colleagues. ${ }^{15}$ Further, because preintervention cardiopulmonary resuscitation (CPR) was much more common in the SE than the CBT series (see Results), and the mortality in patients requiring CPR was much higher, we evaluated the independent effect of SE while controlling for the effect of CPR in both simple stratified and adjusted mixed-effects models. Studies with fewer than 10 subjects were excluded from the regression analysis, and all models were weighted inversely to the variance of each study. A continuity correction was applied to allow for the inclusion of 0 -event studies. Random intercepts were computed for study. All computations were performed using R (R Foundation for Statistical Computing, Vienna, Austria) and SAS software (SAS Institute Inc, Cary NC).

\section{RESULTS}

\section{Search Results}

Using the search strategy outlined in the Methods section, 34 publications of SE outcomes and 41 publications of CBT outcomes were identified; these are listed in Tables 1 and 2, respectively. In the SE group, the mean \pm standard deviation of patients per study was $32.4 \pm 36.6$ (median, 21 patients per study). In the CBT group, the mean \pm standard deviation of patients per study was $40.2 \pm 39.4$ (median, 25 patients per study).

\section{Outcomes of CBT}

Overall, a total of 1650 patients across 41 publications underwent CBT. A breakdown of the different CBT approaches is depicted in Figure 2. Of note, a majority of publications (23 out of 41) utilized more than 1 CBT 
TABLE 1. Studies included for catheter-based therapies. Studies in boldface type are included in Figure 3

\begin{tabular}{|c|c|c|c|c|c|c|c|c|c|c|c|}
\hline $\begin{array}{c}\text { Serial } \\
\text { no. }\end{array}$ & Authors & Year & $\begin{array}{l}\text { No. of } \\
\text { patients }\end{array}$ & $\begin{array}{l}\text { Massive } \\
\text { PE }\end{array}$ & $\begin{array}{c}\text { Submassive } \\
\text { PE }\end{array}$ & CPR & $\begin{array}{l}\text { Procedural } \\
\text { success }\end{array}$ & $\begin{array}{c}\text { Mortality } \\
\text { overall }\end{array}$ & $\begin{array}{c}\text { Mortality } \\
\text { without } \\
\text { CPR }\end{array}$ & $\begin{array}{c}\text { Mortality } \\
\text { with } \\
\text { CPR }\end{array}$ & Technique \\
\hline 1 & $\begin{array}{c}\text { Bloomer and } \\
\text { colleagues }^{16}\end{array}$ & 2017 & 137 & $16(11.7)$ & $121(88.3)$ & ND & ND & $5(3.6)$ & ND & ND & $\begin{array}{l}\text { Both standard and } \\
\text { ultrasound-assisted } \\
\text { catheter directed } \\
\text { thrombolysis }\end{array}$ \\
\hline 2 & $\begin{array}{l}\text { Liang and } \\
\text { colleagues }^{17}\end{array}$ & 2016 & 63 & 8 (12.7) & $55(87.3)$ & ND & $58(92.1)$ & $2(3.2)$ & ND & ND & $\begin{array}{l}\text { Both standard and } \\
\text { ultrasound assisted } \\
\text { catheter directed } \\
\text { thrombolysis }\end{array}$ \\
\hline 3 & $\begin{array}{l}\text { Sag and } \\
\text { colleagues }^{18}\end{array}$ & 2016 & 13 & 13 (100) & $0(0 \%)$ & $1(7.7)$ & ND & $0(0)$ & $0(0)$ & $0(0)$ & $\begin{array}{l}\text { Ultrasound-assisted } \\
\text { catheter-directed } \\
\text { thrombolysis }\end{array}$ \\
\hline 4 & $\begin{array}{l}\text { Kabhrel and } \\
\text { colleagues }{ }^{19}\end{array}$ & 2016 & 28 & $14(50)$ & $14(50)$ & ND & ND & ND & ND & ND & $\begin{array}{c}\text { Catheter-directed } \\
\text { thrombolysis }\end{array}$ \\
\hline 5 & $\begin{array}{l}\text { Yoo and } \\
\text { colleagues }^{20}\end{array}$ & 2016 & 28 & $12(42.9)$ & $16(57.1)$ & $5(17.8)$ & ND & $4(14.3)$ & ND & ND & $\begin{array}{l}\text { Catheter-directed } \\
\text { thrombolysis }+ \\
\text { mechanical disruption }\end{array}$ \\
\hline 6 & $\begin{array}{c}\text { Dilektasli and } \\
\text { colleagues }^{21}\end{array}$ & 2016 & 15 & $6(40)$ & $9(60)$ & $0(0)$ & $14(93.3)$ & $2(13.3)$ & $2(100)$ & $0(0)$ & $\begin{array}{l}\text { Catheter-directed } \\
\text { thrombolysis }+ \\
\text { aspiration embolectomy }\end{array}$ \\
\hline 7 & $\begin{array}{l}\text { Piazza and } \\
\text { colleagues }^{22}\end{array}$ & 2015 & 150 & $31(20.7)$ & $119(79.3)$ & ND & ND & $3(2)$ & ND & ND & $\begin{array}{l}\text { Ultrasound-assisted } \\
\text { catheter-directed } \\
\text { thrombolysis }\end{array}$ \\
\hline 8 & $\begin{array}{l}\text { George and } \\
\text { colleagues }^{23}\end{array}$ & 2015 & 32 & ND & ND & ND & ND & $2(6.3)$ & ND & ND & $\begin{array}{c}\text { Catheter-directed } \\
\text { thrombolysis }\end{array}$ \\
\hline 9 & $\begin{array}{l}\text { Kuo and } \\
\text { colleagues }^{24}\end{array}$ & 2015 & 101 & $28(28)$ & $73(72)$ & ND & $90(89)$ & $6(6)$ & ND & ND & $\begin{array}{l}\text { Catheter-directed } \\
\text { mechanical } \\
\text { embolectomy } \\
\text { or thrombolysis }\end{array}$ \\
\hline 10 & $\begin{array}{l}\text { Engelberger } \\
\text { and } \\
\text { colleagues }^{25}\end{array}$ & 2015 & 52 & $14(27)$ & $38(73)$ & $3(6)$ & 49 (94) & $2(4)$ & $0(0)$ & $2(100)$ & $\begin{array}{l}\text { Ultrasound-assisted } \\
\text { catheter-directed } \\
\text { thrombolysis }\end{array}$ \\
\hline 11 & $\begin{array}{l}\text { Dumantepe and } \\
\text { colleagues }^{26}\end{array}$ & 2015 & 36 & $11(31)$ & $25(69)$ & ND & $35(97.2)$ & $2(5.5)$ & ND & ND & $\begin{array}{l}\text { Mechanical aspiration } \\
\text { embolectomy }\end{array}$ \\
\hline 12 & $\begin{array}{c}\text { Quintana and } \\
\text { colleagues }^{27}\end{array}$ & 2014 & 10 & $2(20)$ & $8(80)$ & $0(0)$ & $10(100)$ & $0(0)$ & $0(0)$ & $0(0)$ & $\begin{array}{l}\text { Ultrasound-assisted } \\
\text { catheter-directed } \\
\text { thrombolysis }\end{array}$ \\
\hline 13 & $\begin{array}{l}\text { Mohan and } \\
\text { colleagues }^{28}\end{array}$ & 2014 & 50 & $20(40)$ & $30(60)$ & $0(0)$ & $46(92)$ & $2(4)$ & $2(100)$ & $0(0)$ & $\begin{array}{l}\text { Mechanical disruption } \\
\text { plus catheter-directed } \\
\text { thrombolysis }\end{array}$ \\
\hline 14 & $\begin{array}{l}\text { Gaba and } \\
\text { colleagues }^{29}\end{array}$ & 2014 & 19 & $4(21)$ & $15(79)$ & $0(0)$ & $18(95)$ & $1(5)$ & $1(100)$ & $0(0)$ & $\begin{array}{l}\text { Catheter-directed } \\
\text { thrombolysis }\end{array}$ \\
\hline 15 & $\begin{array}{l}\text { Akin and } \\
\text { colleagues }^{30}\end{array}$ & 2014 & 17 & $5(29)$ & $12(71)$ & ND & $16(94)$ & $1(6)$ & ND & ND & $\begin{array}{l}\text { Catheter-directed } \\
\text { thrombolysis }\end{array}$ \\
\hline 16 & $\begin{array}{l}\text { Kennedy and } \\
\text { colleagues }^{31}\end{array}$ & 2013 & 60 & $12(20)$ & $48(80)$ & $1(2)$ & $59(98)$ & $3(5)$ & $3(100)$ & $0(0)$ & $\begin{array}{l}\text { Ultrasound-assisted } \\
\text { catheter-directed } \\
\text { thrombolysis }\end{array}$ \\
\hline 17 & $\begin{array}{l}\text { Bonvini and } \\
\text { colleagues }^{32}\end{array}$ & 2013 & 10 & $10(100)$ & $0(0)$ & $6(60)$ & $8(80)$ & $7(70)$ & $3(42.8)$ & $4(57.2)$ & $\begin{array}{l}\text { Rheolytic } \\
\text { embolectomy }\end{array}$ \\
\hline
\end{tabular}


TABLE 1. Continued

\begin{tabular}{|c|c|c|c|c|c|c|c|c|c|c|c|}
\hline $\begin{array}{c}\text { Serial } \\
\text { no. }\end{array}$ & Authors & Year & $\begin{array}{l}\text { No. of } \\
\text { patients }\end{array}$ & $\begin{array}{c}\text { Massive } \\
\text { PE }\end{array}$ & $\begin{array}{c}\text { Submassive } \\
\text { PE }\end{array}$ & CPR & $\begin{array}{c}\text { Procedural } \\
\text { success }\end{array}$ & $\begin{array}{l}\text { Mortality } \\
\text { overall }\end{array}$ & $\begin{array}{c}\text { Mortality } \\
\text { without } \\
\text { CPR }\end{array}$ & $\begin{array}{l}\text { Mortality } \\
\text { with } \\
\text { CPR }\end{array}$ & Technique \\
\hline 18 & $\begin{array}{l}\text { Nassiri and } \\
\text { colleagues }^{33}\end{array}$ & 2012 & 15 & $1(7)$ & $14(93)$ & $1(7)$ & $9(60)$ & $0(0)$ & $0(0)$ & $0(0)$ & Rheolytic embolectomy \\
\hline 19 & $\begin{array}{l}\text { Cuculi and } \\
\text { colleagues }^{34}\end{array}$ & 2012 & 63 & $17(27)$ & $46(73)$ & 3 & 57 & 4 & ND & ND & $\begin{array}{l}\text { Aspiration embolectomy } \\
\text { plus catheter-directed } \\
\text { thrombolysis }\end{array}$ \\
\hline 20 & $\begin{array}{l}\text { Liu and } \\
\text { colleagues }^{35}\end{array}$ & 2011 & 14 & $5(36)$ & $9(64)$ & $0(0)$ & $14(100)$ & $0(0)$ & $0(0)$ & $0(0)$ & $\begin{array}{l}\text { Aspiration/mechanical } \\
\text { disruption plus } \\
\text { catheter-directed } \\
\text { thrombolysis }\end{array}$ \\
\hline 21 & $\begin{array}{l}\text { Gao and } \\
\text { colleagues }^{36}\end{array}$ & 2011 & 46 & $11(24)$ & $35(76)$ & $0(0)$ & $46(100)$ & $0(0)$ & $0(0)$ & $0(0)$ & $\begin{array}{l}\text { Aspiration embolectomy } \\
\text { plus catheter-directed } \\
\text { thrombolysis; mechanical } \\
\text { disruption if aspiration } \\
\text { failed }\end{array}$ \\
\hline 22 & $\begin{array}{c}\text { Ferrigino and } \\
\text { colleagues }^{37}\end{array}$ & 2011 & 16 & $5(31)$ & $11(69)$ & $1(6)$ & $16(100)$ & $1(6)$ & $0(0)$ & $1(100)$ & $\begin{array}{l}\text { Rheolytic embolectomy } \\
\text { plus catheter-directed } \\
\text { thrombolysis }\end{array}$ \\
\hline 23 & $\begin{array}{l}\text { De Gregorio } \\
\text { and } \\
\text { colleagues }^{38}\end{array}$ & 2011 & 111 & $111(100)$ & $0(0)$ & $0(0)$ & $111(100)$ & $4(4)$ & $4(100)$ & $0(0)$ & $\begin{array}{l}\text { Mechanical disruption } \\
\text { plus catheter-directed } \\
\text { thrombolysis }\end{array}$ \\
\hline 24 & $\begin{array}{c}\text { Yamamoto and } \\
\text { colleagues }^{39}\end{array}$ & 2009 & 50 & $12(24)$ & $38(76)$ & $5(10)$ & $50(100)$ & $3(6)$ & ND & ND & $\begin{array}{l}\text { Catheter-directed } \\
\text { thrombolysis }+/- \\
\text { either mechanical } \\
\text { disruption or aspiration } \\
\text { embolectomy }\end{array}$ \\
\hline 25 & $\begin{array}{l}\text { Lin and } \\
\quad \text { colleagues }\end{array}$ & 2009 & 25 & ND & ND & ND & $20 / 25(80)$ & $3(12)$ & ND & ND & $\begin{array}{l}\text { Ultrasound-assisted } \\
\text { or standard catheter- } \\
\text { directed thrombolysis }\end{array}$ \\
\hline 26 & $\begin{array}{l}\text { Chechi and } \\
\text { colleagues }^{41}\end{array}$ & 2009 & 51 & $22(43)$ & $29(57)$ & $0(0)$ & $47(92)$ & $8(16)$ & $8(100)$ & $0(0)$ & $\begin{array}{l}\text { Rheolytic embolectomy } \\
\text { plus catheter-directed } \\
\text { thrombolysis }\end{array}$ \\
\hline 27 & $\begin{array}{l}\text { Spies and } \\
\text { colleagues }^{42}\end{array}$ & 2008 & 13 & $13(100)$ & $0(0)$ & ND & $12(92)$ & $2(15)$ & ND & ND & Rheolytic embolectomy \\
\hline 28 & $\begin{array}{l}\text { Nakazawa } \\
\text { and } \\
\text { colleagues }^{43}\end{array}$ & 2008 & 25 & ND & ND & ND & $18(72)$ & 0() & $0(0)$ & $0(0)$ & $\begin{array}{l}\text { Mechanical disruption } \\
\text { and aspiration } \\
\text { embolectomy plus } \\
\text { catheter-directed } \\
\text { thrombolysis }\end{array}$ \\
\hline 29 & $\begin{array}{l}\text { Margheri and } \\
\text { colleagues }^{44}\end{array}$ & 2008 & 25 & $20(80)$ & $5(20)$ & ND & $25(100)$ & $4(16)$ & ND & ND & Rheolytic embolectomy \\
\hline 30 & $\begin{array}{l}\text { Kuo and } \\
\text { colleagues }^{45}\end{array}$ & 2008 & 12 & $12(100)$ & $0(0)$ & $0(0)$ & $10(83)$ & $2(17)$ & $2(100)$ & $0(0)$ & $\begin{array}{l}\text { Mechanical disruption } \\
\text { and aspiration } \\
\text { embolectomy plus } \\
\text { catheter-directed } \\
\text { thrombolysis; rheolytic } \\
\text { treatment if failed }\end{array}$ \\
\hline
\end{tabular}

(Continued) 
TABLE 1. Continued

\begin{tabular}{|c|c|c|c|c|c|c|c|c|c|c|c|}
\hline $\begin{array}{c}\text { Serial } \\
\text { no. }\end{array}$ & Authors & Year & $\begin{array}{l}\text { No. of } \\
\text { patients }\end{array}$ & $\begin{array}{c}\text { Massive } \\
\text { PE }\end{array}$ & $\begin{array}{c}\text { Submassive } \\
\text { PE }\end{array}$ & CPR & $\begin{array}{c}\text { Procedural } \\
\text { success }\end{array}$ & $\begin{array}{c}\text { Mortality } \\
\text { overall }\end{array}$ & $\begin{array}{c}\text { Mortality } \\
\text { without } \\
\text { CPR }\end{array}$ & $\begin{array}{l}\text { Mortality } \\
\text { with } \\
\text { CPR }\end{array}$ & Technique \\
\hline 31 & $\begin{array}{l}\text { Eid-Lidt and } \\
\text { colleagues }^{46}\end{array}$ & 2008 & 18 & $8(44)$ & $10(56)$ & $0(0)$ & $16(89)$ & $1(6)$ & $1(100)$ & $0(0)$ & $\begin{array}{l}\text { Mechanical disruption } \\
\text { plus aspiration } \\
\text { embolectomy and } \\
\text { catheter-directed } \\
\text { thrombolysis }\end{array}$ \\
\hline 32 & $\begin{array}{l}\text { Pieri and } \\
\text { colleagues }^{47}\end{array}$ & 2007 & 164 & $164(100)$ & $0(0)$ & $0(0)$ & $138(84)$ & $2(1)$ & $2(100)$ & $0(0)$ & $\begin{array}{l}\text { Mechanical disruption } \\
\text { plus catheter-directed } \\
\text { thrombolysis }\end{array}$ \\
\hline 33 & $\begin{array}{c}\text { Chauhan and } \\
\text { colleagues }^{48}\end{array}$ & 2007 & 14 & $6(43)$ & $8(57)$ & $0(0)$ & $12(86)$ & $3(21)$ & $3(100)$ & $0(0)$ & Rheolytic embolectomy \\
\hline 34 & $\begin{array}{l}\text { Barbosa and } \\
\text { colleagues }^{49}\end{array}$ & 2007 & 10 & $10(100)$ & $0(0)$ & $1(10)$ & $9(90)$ & $1(105)$ & $0(0)$ & $1(100)$ & Mechanical disruption \\
\hline 35 & $\begin{array}{l}\text { Tajima and } \\
\text { colleagues }^{50}\end{array}$ & 2004 & 25 & $6(24)$ & $19(76)$ & $1(4)$ & $25(100)$ & $0(0)$ & $0(0)$ & $0(0)$ & $\begin{array}{l}\text { Mechanical disruption } \\
\text { and aspiration } \\
\text { embolectomy plus } \\
\text { catheter-directed } \\
\text { thrombolysis }\end{array}$ \\
\hline 36 & $\begin{array}{l}\text { Tajima and } \\
\text { colleagues }^{51}\end{array}$ & 2004 & 15 & ND & ND & ND & $15(100)$ & $0(0)$ & $0(0)$ & $0(0)$ & $\begin{array}{l}\text { Percutaneous manual } \\
\text { aspiration embolectomy }\end{array}$ \\
\hline 37 & $\begin{array}{l}\text { Zeni and } \\
\text { colleagues }^{52}\end{array}$ & 2003 & 17 & $17(100)$ & $0(0)$ & $0(0)$ & $16(94)$ & $2(12)$ & $2(100)$ & $0(0)$ & $\begin{array}{l}\text { Rheolytic embolectomy } \\
\text { plus catheter-directed } \\
\text { thrombolysis }\end{array}$ \\
\hline 38 & $\begin{array}{l}\text { De Gregorio } \\
\text { and } \\
\text { colleagues }^{53}\end{array}$ & 2002 & 59 & $59(100)$ & $0(0)$ & $0(0)$ & $56(95)$ & $3(5)$ & $3(100)$ & $0(0)$ & $\begin{array}{l}\text { Mechanical disruption } \\
\text { plus catheter-directed } \\
\text { thrombolysis }\end{array}$ \\
\hline 39 & $\begin{array}{l}\text { Schmitz-Rode } \\
\text { and } \\
\text { colleagues }^{54}\end{array}$ & 2000 & 20 & $20(100)$ & $0(0)$ & $7(35)$ & $16(80)$ & $4(20)$ & $1(25)$ & $3(75)$ & $\begin{array}{l}\text { Mechanical disruption } \\
\text { plus catheter-directed } \\
\text { thrombolysis }\end{array}$ \\
\hline 40 & $\begin{array}{l}\text { Fava and } \\
\text { colleagues }^{55}\end{array}$ & 2000 & 11 & 7 (64) & $4(36)$ & $1(9)$ & $10(91)$ & $1(14)$ & $0(0)$ & $1(100)$ & $\begin{array}{l}\text { Rheolytic/aspiration } \\
\text { embolectomy plus } \\
\text { catheter-directed } \\
\text { thrombolysis }\end{array}$ \\
\hline 41 & $\begin{array}{l}\text { Schmitz-Rode and } \\
\text { colleagues }^{56}\end{array}$ & 1998 & 10 & 10 (100) & $0(0)$ & $3(30)$ & $6(60)$ & $2(20)$ & $1(50)$ & $1(50)$ & $\begin{array}{l}\text { Mechanical disruption } \\
\text { plus catheter-directed } \\
\text { thrombolysis }\end{array}$ \\
\hline
\end{tabular}

Values are presented as n (\%). PE, Pulmonary embolism; $C P R$, cardiopulmonary resuscitation; $N D$, no data.

approach to treat individual patients, to achieve therapeutic efficacy. Thirty-seven of 41 CBT publications (90\%) provided information regarding $\mathrm{PE}$ classification. Of the 1553 patients in these studies, $742(47.8 \%)$ had massive $\mathrm{PE}$, and $811(52.2 \%)$ had submassive PE. Twenty-eight of 41 CBT publications $(68.3 \%)$ provided information regarding pretreatment CPR. Within these pre-CBT CPR publications, 596 out of $983(60.6 \%)$ had massive PE, whereas 387 out of $983(39.4 \%)$ had submassive PE. Twenty-seven of 41 studies $(65.8 \%)$ provided stratification of mortality based on CPR status. Mortality in the CPR group was 13 out of $25(52.0 \%)$ compared with 38 out of $857(4.4 \%)$ in the cohort without pre-CBT CPR. Twentyfive of 41 publications $(61.0 \%)$ provided information both on PE classification and pretreatment CPR mortality classification. Of 842 patients in these studies, 25 (3.0\%) required pre-CBT CPR. Mortality within pre-CBT CPR group was much higher: 13 out of $25(52.0 \%)$ compared with 38 out of $817(4.6 \%)$ in the group without pre-CBT CPR.

Procedural success (ie, technical adequacy of CBT determined by the authors as assessed either by angiographic or physiologic criteria) was reported in 35 out of 41 studies. Technically satisfactory outcomes were noted in 1157 of $1262(91.7 \%)$ patients; that is, the failure rate was $8.3 \%$.

One publication did not report mortality outcomes. Hospital mortality of the entire CBT group thus was 92 out of $1622(5.7 \%)$. As in the SE group, mortality was 
TABLE 2. Studies included for surgical embolectomy. Studies in boldface are included in Figure 3

\begin{tabular}{|c|c|c|c|c|c|c|c|c|c|c|}
\hline $\begin{array}{c}\text { Serial } \\
\text { no. }\end{array}$ & Authors & Year & $\begin{array}{c}\text { No. of } \\
\text { patients }\end{array}$ & $\begin{array}{c}\text { Massive } \\
\text { PE }\end{array}$ & $\begin{array}{c}\text { Submassive } \\
\text { PE }\end{array}$ & $\begin{array}{c}\text { Uncomplicated } \\
\text { PE } \\
\end{array}$ & CPR & $\begin{array}{c}\text { Mortality } \\
\text { overall }\end{array}$ & $\begin{array}{c}\text { Mortality } \\
\text { without } \\
\text { CPR } \\
\end{array}$ & $\begin{array}{c}\text { Mortality } \\
\text { with } \\
\text { CPR } \\
\end{array}$ \\
\hline 1 & Lehrnet and colleagues $^{57}$ & 2017 & 50 & $28(56)$ & $22(44)$ & $0(0)$ & $12(24)$ & $4(8)$ & ND & ND \\
\hline 2 & Edelman and colleagues ${ }^{58}$ & 2016 & 37 & $20(54.1)$ & $17(45.9)$ & $0(0)$ & $13(35.1)$ & $2(5.4)$ & ND & ND \\
\hline 3 & Cho and colleagues ${ }^{59}$ & 2016 & 26 & $26(100)$ & $0(0)$ & $0(0)$ & $11(42.3)$ & $4(15.4)$ & ND & ND \\
\hline 4 & Keeling and colleagues ${ }^{60}$ & 2016 & 214 & 38 (17.8) & $176(82.2)$ & $0(0)$ & $28(13.1)$ & $25(11.7)$ & $16(7.5)$ & $9(4.2)$ \\
\hline 5 & Neely and colleagues ${ }^{61}$ & 2015 & 105 & $49(47)$ & $56(53)$ & $0(0)$ & $11(10)$ & $8(8)$ & $3(38)$ & $5(62)$ \\
\hline 6 & Azari and colleagues ${ }^{62}$ & 2015 & 30 & $30(100)$ & $0(0)$ & $0(0)$ & $12(40)$ & $5(17)$ & ND & ND \\
\hline 7 & Worku and colleagues ${ }^{63}$ & 2014 & 20 & $12(60)$ & $8(40)$ & $0(0)$ & $3(15)$ & $1(5)$ & $1(100)$ & $0(0)$ \\
\hline 8 & Osborne and colleagues ${ }^{64}$ & 2014 & 15 & $12(80)$ & $3(20)$ & $0(0)$ & $2(13)$ & $2(13)$ & $0(0)$ & $2(100)$ \\
\hline 9 & Wu and colleagues ${ }^{65}$ & 2013 & 25 & $16(64)$ & $7(28)$ & $2(8)$ & $8(32)$ & $5(20)$ & $1(20)$ & $4(80)$ \\
\hline 10 & Aymard and colleagues ${ }^{66}$ & 2013 & 39 & ND & ND & $0(0)$ & ND & $4(10)$ & ND & ND \\
\hline 11 & Taniquchi and colleagues $^{67}$ & 2012 & 32 & $26(81)$ & $6(19)$ & $0(0)$ & $3(9)$ & $6(19)$ & $5(83)$ & $1(17)$ \\
\hline 12 & Takahashi and colleagues ${ }^{68}$ & 2012 & 24 & $16(67)$ & $8(33)$ & $0(0)$ & $11(46)$ & $3(13)$ & $0(0)$ & $3(100)$ \\
\hline 13 & Marshall and colleagues $^{69}$ & 2012 & 10 & $10(100)$ & $0(0)$ & $0(0)$ & $6(60)$ & $4(40)$ & $1(25)$ & $3(75)$ \\
\hline 14 & Malekan and colleagues $^{70}$ & 2012 & 26 & $7(27)$ & $19(73)$ & $0(0)$ & $0(0)$ & $0(0)$ & $0(0)$ & $0(0)$ \\
\hline 15 & Zarrabi and colleagues $^{71}$ & 2011 & 30 & $11(37)$ & $19(63)$ & $0(0)$ & $3(10)$ & $2(7)$ & $1(50)$ & $1(50)$ \\
\hline 16 & Greelish and colleagues ${ }^{72}$ & 2011 & 15 & $7(47)$ & $8(53)$ & $0(0)$ & $2(13)$ & $2(13)$ & $0(0)$ & $2(100)$ \\
\hline 17 & Fukuda and colleagues $^{73}$ & 2011 & 19 & $17(90)$ & $2(10)$ & $0(0)$ & $4(21)$ & $1(5)$ & $1(33)$ & $0(67)$ \\
\hline 18 & Vohra and colleagues $^{74}$ & 2010 & 21 & $21(100)$ & $0(0)$ & $0(0)$ & $9(43)$ & $4(19)$ & ND & ND \\
\hline 19 & $\begin{array}{l}\text { Sareyyupoqlu and } \\
\text { colleagues }^{75}\end{array}$ & 2010 & 18 & $12(67)$ & $6(3)$ & $0(0)$ & $7(39)$ & $4(22)$ & $0(0)$ & $4(25)$ \\
\hline 20 & Carvalho and colleagues ${ }^{76}$ & 2010 & 16 & $16(100)$ & $0(0)$ & $0(0)$ & $7(44)$ & $7(44)$ & $1(14)$ & $6(86)$ \\
\hline 21 & Kukla and colleagues ${ }^{77}$ & 2009 & 15 & $6(40)$ & $9(60)$ & $0(0)$ & ND & $5(33)$ & ND & ND \\
\hline 22 & Sadaba and colleagues ${ }^{78}$ & 2008 & 20 & $9(45)$ & $11(55)$ & $0(0)$ & $1(5)$ & $2(10)$ & $1(50)$ & $1(50)$ \\
\hline 23 & Kadner and colleagues $^{79}$ & 2008 & 25 & $25(100)$ & $0(0)$ & $0(0)$ & $8(32)$ & $2(8)$ & $0(0)$ & $2(100)$ \\
\hline 24 & Ahmed and colleagues ${ }^{80}$ & 2008 & 15 & 7 (46.7) & $6(40.0)$ & $2(13.3)$ & $0(0)$ & $3(20)$ & $3(100)$ & $0(0)$ \\
\hline 25 & Diggonet and colleagues $^{81}$ & 2007 & 21 & $14(67)$ & $7(33)$ & $0(0)$ & $6(29)$ & $8(38)$ & $4(50)$ & $4(50)$ \\
\hline 26 & $\begin{array}{l}\text { Amirghofran and } \\
\text { colleagues }^{82}\end{array}$ & 2007 & 11 & $11(100)$ & $0(0)$ & $0(0)$ & $3(27)$ & $2(18)$ & $1(50)$ & $1(50)$ \\
\hline 27 & Spagnolo and colleagues ${ }^{83}$ & 2006 & 21 & $21(100)$ & $0(0)$ & $0(0)$ & $2(10)$ & $0(0)$ & $0(0)$ & $0(0)$ \\
\hline 28 & Meneveau and colleagues ${ }^{84}$ & 2006 & 14 & $14(100)$ & $0(0)$ & $0(0)$ & ND & $1(7)$ & ND & ND \\
\hline 29 & Sukhija and colleagues ${ }^{85}$ & 2005 & 18 & $18(100)$ & $0(0)$ & $0(0)$ & ND & $2(11)$ & ND & ND \\
\hline 30 & Leachhe and colleagues ${ }^{86}$ & 2005 & 47 & $28(59.6)$ & $15(31.9)$ & $4(8.5)$ & $6(13)$ & $3(6)$ & $2(67)$ & $1(33)$ \\
\hline 31 & Dauphine and colleagues ${ }^{87}$ & 2005 & 11 & $11(100)$ & $0(0)$ & $0(0)$ & $4(36)$ & $3(27)$ & $0(0)$ & $3(100)$ \\
\hline 32 & $\begin{array}{l}\text { Yalamanchili and } \\
\text { colleagues }^{88}\end{array}$ & 2004 & 13 & $4(31)$ & $9(69)$ & $0(0)$ & $2(15)$ & $1(8)$ & $0(0)$ & $1(100)$ \\
\hline 33 & Ullmann and colleagues ${ }^{89}$ & 1999 & 40 & ND & ND & $0(0)$ & $19(48)$ & $14(35)$ & $2(14)$ & $12(86)$ \\
\hline 34 & Doerge and colleagues ${ }^{90}$ & 1999 & 41 & $33(80)$ & $8(20)$ & $0(0)$ & $14(34)$ & $12(29)$ & $3(25)$ & $9(75)$ \\
\hline
\end{tabular}

Values are presented as n (\%). $P E$, Pulmonary embolism; $C P R$, cardiopulmonary resuscitation; $N D$, no data.

not classified based on PE classification. Mortality was classified based on pre-CBT CPR status; of 28 publications providing pretreatment CPR information, 25 classified mortality based on it. Within these publications, hospital mortality was 51 out of $842(6.1 \%)$. Mortality was 13 out of $25(52.0 \%)$ in those patients with pre-CBT CPR, whereas it was 38 out of $764(5.0 \%)$ in those patients without pre-CBT CPR.

For the meta-analysis, no CBT study had 10 or more patients who received CPR, so no pooled estimates were 


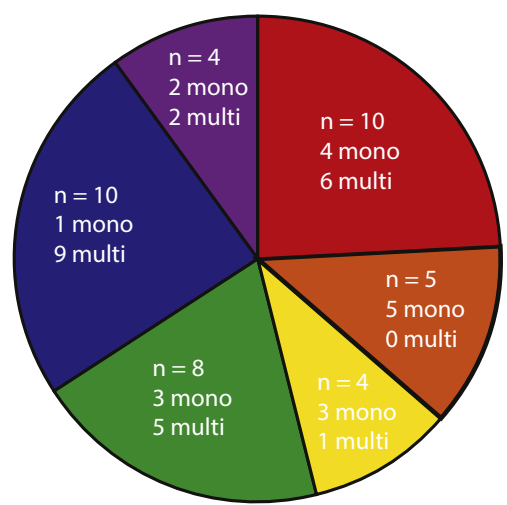

- Standard catheter-directed thrombolysis Ultrasound catheter-directed thrombolysis

Mixture of types of catheter-directed thrombolysis in different patients

Rheolytic catheter-based disruption

- Directly mechanical catheter-based disruption

- Catheter-based aspiration

FIGURE 2. Representation of different types of catheter-based therapies (CBTs) in the CBT studies analyzed. Data are presented as a pie chart, with the indicated rainbow color legend. For a given type of primary CBT, studies in which the primary CBT was used exclusively as a single modality in patients are indicated as "mono." Studies in which the primary CBT was used as a first-line therapy, but as part of a strategy in which more than 1 type of CBT was used within individual patients, are indicated as "multi." Studies denoted as "multi" had individual patients who each received more than 1 type of CBT, but the first type of CBT used denoted as the primary therapy. Consequently, for a given type of primary CBT, mono and multi add up to the total number of studies for that type of primary CBT. The category in yellow refers to studies in which different types of primary CBT were reported; for 3 articles, several patients within a given study in this category had standard catheter-directed or ultrasound-assisted catheterdirected thrombolysis, but not a combination of the 2 . In 1 article, 2 different types of CBT were employed, and some patients received an additional modality of CBT (also denoted as "multi").

computed for CBT-CPR. The overall weighted random effects estimate for CBT was 6\% (95\% confidence interval [CI], 4\%-9\%) (Figure 3).

\section{Outcomes of SE}

Overall, a total of 1101 patients across 34 publications underwent SE. Thirty-one of 34 publications (91.1\%) provided information regarding $\mathrm{PE}$ classification (massive, submassive, or uncomplicated). Of 975 patients in these studies, 545 (55.9\%) had massive PE, 422 (43.3\%) had submassive PE, and $8(0.8 \%)$ had uncomplicated PE. Thirty of 34 publications $(88.2 \%)$ provided information regarding pretreatment CPR; of 1015 patients in these studies, 217 $(21.4 \%)$ required pre-SE CPR. Procedural success (ie, technical adequacy of embolectomy) generally was not reported.

Hospital mortality of the entire SE group was 154 out of $1101(14.0 \%)$. In the publications reporting PE classification, mortality generally was not categorized based on it. However, mortality was classified based on pre-SE CPR status when available; of 30 publications providing pretreatment CPR information, 25 classified mortality based on it. Within these publications, hospital mortality was 120 out of $834(14.0 \%)$. Mortality was 74 out of 160 $(46.3 \%)$ in those patients with pre-SE CPR, whereas it was 46 out of $674(6.8 \%)$ in those patients without pre-SE CPR. Twenty-four of 34 publications (70.6\%) provided information both on $\mathrm{PE}$ classification and pretreatment CPR mortality classification. Within these publications, 412 out of $794(52.0 \%)$ had massive PE, 374 out of $794(47.1 \%)$ had submassive PE, whereas 8 out of $794(0.9 \%)$ had uncomplicated PE. Hospital mortality was 106 out of $794(13.4 \%)$. Mortality was 62 out of141 $(44.0 \%)$ in those patients with pre-SE CPR, whereas it was 44 out of $653(6.7 \%)$ in those patients without pre-SE CPR. In SE patients not receiving $\mathrm{CPR}$, the pooled random effect estimate of mortality was $9 \%(95 \% \mathrm{CI}, 6 \%-11 \%)$. This was not statistically significantly different from CBT without CPR $(P=.14)$ (Figure 3).

The shift in the percentages of PE types in the SE group when only the selected subgroup of publications (those that both classified PE type and stratified mortality based on CPR status) is considered, particularly relative to the analogous CBT subgroup, is solely attributable to a single outlier study of Keeling and colleagues (discussed in detail below), ${ }^{24}$ the single largest SE study, with a ratio of massive to submassive $\mathrm{PE}<1: 4$. If this study were excluded, of the remaining 23 studies, of 580 patients, $374(64.5 \%)$ had massive PE, $198(34.1 \%)$ had submassive PE and 8 $(1.4 \%)$ had uncomplicated PE.

\section{Effect of CPR and CPR Status on Differential Mortality Post-CBT Versus SE}

As outlined in the Methods section, we conducted a statistical analysis of the effects of CPR status on the mortality of CBT and SE. It is important to note that in these statistical models, only the studies that had data regarding the presence or absence of CPR, and were further stratified based on mortality, were analyzed. Moderate heterogeneity between the studies was identified, as indicated by $I^{2}=18 \%$. Mixed-model adjustment and inverse-variance weighting was used to estimate the adjusted odds ratio (OR) effects. The adjusted OR for CPR in a random effects model with treatment considered was 9.79 (95\% CI, 4.98-19.17; $P<.0001)$. However, as discussed above, 217 out of $1015 \mathrm{SE}$ patients $(21.4 \%)$ had a history of CPR, in comparison to 38 out of 983 CBT patients (4.0\%). The adjusted OR for mortality for SE versus CBT was 1.36 (95\% CI, 0.80-2.32). As a result, when controlling for the higher risk of the SE population, SE and CBT were found to have mortality risks that were not statistically significantly different $(P=.84)$. Thus, even without individual-level risk adjustment, this analysis shows that 


\begin{tabular}{|c|c|c|c|c|c|c|}
\hline $\begin{array}{l}\text { Study or } \\
\text { Subgroup } \\
\text { Group = CBT }\end{array}$ & Events & Total & $\begin{array}{l}\text { Weight } \\
\text { (fixed) }\end{array}$ & $\begin{array}{r}\text { Weight } \\
\text { (random) }\end{array}$ & IV, Fixed + Random, 95\% CI & IV, Fixed + Random, $95 \% \mathrm{CI}$ \\
\hline Engelberger CBT 10 & 0 & 49 & $0.5 \%$ & $1.2 \%$ & $0.00[0.00 ; 0.07]$ & ־ \\
\hline Gao CBT 21 & 0 & 46 & $0.5 \%$ & $1.2 \%$ & $0.00[0.00 ; 0.08]$ & - \\
\hline Piere CBT 32 & 2 & 164 & $2.0 \%$ & $2.5 \%$ & $0.01[0.00 ; 0.04]$ & \pm \\
\hline Nakazawa CBT 28 & 0 & 25 & $0.5 \%$ & $1.2 \%$ & $0.00[0.00 ; 0.14]$ & 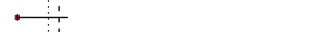 \\
\hline Tajima CBT 35 & 0 & 24 & $0.5 \%$ & $1.2 \%$ & $0.00[0.00 ; 0.14]$ & 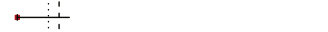 \\
\hline Ferrigino CBT 22 & 0 & 15 & $0.5 \%$ & $1.2 \%$ & $0.00[0.00 ; 0.22]$ & \\
\hline Nassisi CBT 18 & 0 & 15 & $0.5 \%$ & $1.2 \%$ & $0.00[0.00 ; 0.22]$ & \\
\hline Liu CBT 20 & 0 & 14 & $0.5 \%$ & $1.2 \%$ & $0.00[0.00 ; 0.23]$ & \\
\hline Tajima CBT 36 & 0 & 14 & $0.5 \%$ & $1.2 \%$ & $0.00[0.00 ; 0.23]$ & 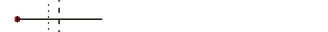 \\
\hline DeGregorio CBT 23 & 4 & 111 & $4.0 \%$ & $2.9 \%$ & $0.04[0.01 ; 0.09]$ & \#- \\
\hline Sag CBT 3 & 0 & 12 & $0.5 \%$ & $1.2 \%$ & $0.00[0.00 ; 0.26]$ & 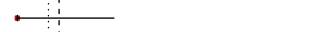 \\
\hline Mohan CBT 13 & 2 & 50 & $2.0 \%$ & $2.4 \%$ & $0.04[0.00 ; 0.14]$ & 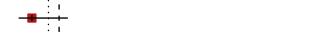 \\
\hline Fava CBT 40 & 0 & 10 & $0.5 \%$ & $1.2 \%$ & $0.00[0.00 ; 0.31]$ & \\
\hline Quintana CBT 12 & 0 & 10 & $0.5 \%$ & $1.2 \%$ & $0.00[0.00 ; 0.31]$ & 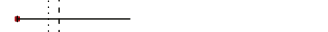 \\
\hline Kennedy CBT 16 & 3 & 60 & $3.0 \%$ & $2.7 \%$ & $0.05[0.01 ; 0.14]$ & 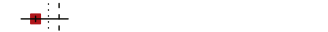 \\
\hline DeGregorio CBT 38 & 3 & 59 & $3.0 \%$ & $2.7 \%$ & $0.05[0.01 ; 0.14]$ & $\rightarrow$ \\
\hline Gaba CBT 14 & 1 & 19 & $1.0 \%$ & $1.8 \%$ & $0.05[0.00 ; 0.26]$ & $\rightarrow$ \\
\hline Eid-Lidt CBT 31 & 1 & 18 & $1.0 \%$ & $1.8 \%$ & $0.06[0.00 ; 0.27]$ & $\rightarrow$ \\
\hline Schmitz-Rode CBT 39 & 1 & 13 & $1.0 \%$ & $1.8 \%$ & $0.08[0.00 ; 0.36]$ & \\
\hline Zeni CBT 37 & 2 & 17 & $1.8 \%$ & $2.4 \%$ & $0.12[0.01 ; 0.36]$ & \\
\hline Dilektasli CBT 6 & 2 & 15 & $1.8 \%$ & $2.4 \%$ & $0.13[0.02 ; 0.40]$ & \\
\hline Chechi CBT 26 & 8 & 51 & $7.0 \%$ & $3.2 \%$ & $0.16[0.07 ; 0.29]$ & \\
\hline Kuo CBT 30 & 2 & 12 & $1.7 \%$ & $2.3 \%$ & $0.17[0.02 ; 0.48]$ & \\
\hline Chauhan CBT 33 & 3 & 14 & $2.4 \%$ & $2.6 \%$ & $0.21[0.05 ; 0.51]$ & \\
\hline Total (fixed effect, 95\% Cl) & & 837 & $37.2 \%$ & -- & $0.07[0.05 ; 0.09]$ & $\bullet$ \\
\hline Total (random effects, $95 \% \mathrm{Cl}$ ) & & & -- & $44.9 \%$ & $0.06[0.04 ; 0.09]$ & 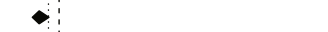 \\
\hline Heterogeneity: $\mathrm{Tau}^{2}=0.1959 ; \mathrm{Chi}^{2}$ & $=29.46, \mathrm{c}$ & $d f=23$ & $(P=0.17)$ & $1^{2}=22 \%$ & & \\
\hline Group = SE-CPR & & & & & & \\
\hline Takahashi SE 12 (+CPR) & 3 & 11 & $2.3 \%$ & $2.5 \%$ & $0.27[0.06 ; 0.61]$ & \\
\hline Keeling SE 4 (+CPR) & 9 & 28 & $6.3 \%$ & $3.2 \%$ & $0.32[0.16 ; 0.52]$ & \\
\hline Neely SE 5 (+CPR) & 5 & 11 & $2.8 \%$ & $2.7 \%$ & $0.45[0.17 ; 0.77]$ & \\
\hline Ullmann SE 33 (+CPR) & 12 & 19 & $4.6 \%$ & $3.0 \%$ & $0.63[0.38 ; 0.84]$ & $\mp$ \\
\hline Doerge SE 34 (+CPR) & 9 & 14 & $3.3 \%$ & $2.8 \%$ & $0.64[0.35 ; 0.87]$ & 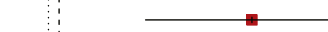 \\
\hline Total (fixed effect, $95 \% \mathrm{CI}$ ) & & 83 & $19.3 \%$ & -- & $0.46[0.35 ; 0.57]$ & \\
\hline Total (random effects, $95 \% \mathrm{Cl}$ ) & & & -- & $14.3 \%$ & $0.47[0.31 ; 0.62]$ & \\
\hline Heterogeneity: $\mathrm{Tau}^{2}=0.2455 ; \mathrm{Chi}^{2}$ & $=7.53, \mathrm{df}$ & $=4(P$ & $=0.11) ; 1^{2}$ & $2=47 \%$ & & \\
\hline Group = SE-no CPR & & & & & & \\
\hline Malekan SE 14 (-CPR) & 0 & 26 & $0.5 \%$ & $1.2 \%$ & $0.00[0.00 ; 0.13]$ & 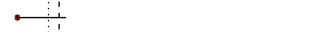 \\
\hline Spagnolo SE 27 (-CPR) & 0 & 19 & $0.5 \%$ & $1.2 \%$ & $0.00[0.00 ; 0.18]$ & 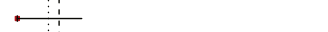 \\
\hline Kadner SE 23 (-CPR) & 0 & 17 & $0.5 \%$ & $1.2 \%$ & $0.00[0.00 ; 0.20]$ & 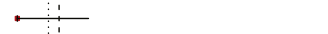 \\
\hline Neely SE 5 (-CPR) & 3 & 94 & $3.0 \%$ & $2.8 \%$ & $0.03[0.01 ; 0.09]$ & \\
\hline Osborne SE 8 (-CPR) & 0 & 13 & $0.5 \%$ & $1.2 \%$ & $0.00[0.00 ; 0.25]$ & \\
\hline Takahashi SE 12 (-CPR) & 0 & 13 & $0.5 \%$ & $1.2 \%$ & $0.00[0.00 ; 0.25]$ & \\
\hline Greelish SE 16 (-CPR) & 0 & 13 & $0.5 \%$ & $1.2 \%$ & $0.00[0.00 ; 0.25]$ & • \\
\hline Zarrabi SE 15 (-CPR) & 1 & 27 & $1.0 \%$ & $1.8 \%$ & $0.04[0.00 ; 0.19]$ & \\
\hline Sareyyupoglu SE 19 (-CPR) & 0 & 11 & $0.5 \%$ & $1.2 \%$ & $0.00[0.00 ; 0.28]$ & 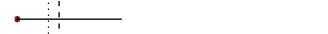 \\
\hline Yalamanchili SE 32 (-CPR) & 0 & 11 & $0.5 \%$ & $1.2 \%$ & $0.00[0.00 ; 0.28]$ & $\longmapsto$ \\
\hline Leachhe SE 30 (-CPR) & 2 & 41 & $2.0 \%$ & $2.4 \%$ & $0.05[0.01 ; 0.17]$ & $\rightarrow$ \\
\hline Sadaba SE 22 (-CPR) & 1 & 19 & $1.0 \%$ & $1.8 \%$ & $0.05[0.00 ; 0.26]$ & $\rightarrow$ \\
\hline Wu SE 9 (-CPR) & 1 & 17 & $1.0 \%$ & $1.8 \%$ & $0.06[0.00 ; 0.29]$ & $\rightarrow$ \\
\hline Worku SE 7 (-CPR) & 1 & 17 & $1.0 \%$ & $1.8 \%$ & $0.06[0.00 ; 0.29]$ & $\rightarrow$ \\
\hline Fukuda SE 15 (-CPR) & 1 & 15 & $1.0 \%$ & $1.8 \%$ & $0.07[0.00 ; 0.32]$ & $\rightarrow$ \\
\hline Keeling SE 4 (-CPR) & 16 & 186 & $15.2 \%$ & $3.5 \%$ & $0.09[0.05 ; 0.14]$ & \\
\hline Ullmann SE (-CPR) & 2 & 21 & $1.9 \%$ & $2.4 \%$ & $0.10[0.01 ; 0.30]$ & i \\
\hline Doerge SE 34 (-CPR) & 3 & 27 & $2.8 \%$ & $2.7 \%$ & $0.11[0.02 ; 0.29]$ & 1 \\
\hline Taniquchi SE 11 (-CPR) & 5 & 29 & $4.3 \%$ & $3.0 \%$ & $0.17[0.06 ; 0.36]$ & \\
\hline Ahmed SE 24 (-CPR) & 3 & 15 & $2.5 \%$ & $2.6 \%$ & $0.20[0.04 ; 0.48]$ & - \\
\hline Diggonet SE 25 (-CPR) & 4 & 15 & $3.0 \%$ & $2.8 \%$ & $0.27[0.08 ; 0.55]$ & \\
\hline Total (fixed effect, $95 \% \mathrm{Cl}$ ) & & 646 & $43.5 \%$ & -- & $0.09[0.06 ; 0.11]$ & 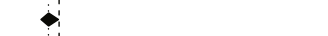 \\
\hline Total (random effects, $95 \% \mathrm{CI}$ ) & & & -- & $40.8 \%$ & $0.09[0.06 ; 0.11]$ & 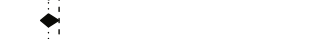 \\
\hline Heterogeneity: $\mathrm{Tau}^{2}=0.0121 ; \mathrm{Chi}^{2}$ & 20.43 & tf & $(P=0.43)$ & $; 1^{2}=2 \%$ & & \\
\hline Total (fixed effect, 95\% Cl) & & 1566 & $100.0 \%$ & -- & $0.11[0.10 ; 0.14]$ & $\bullet$ \\
\hline Total (random effects, $95 \% \mathrm{Cl}$ ) & & & -- & $100.0 \%$ & $0.09[0.06 ; 0.12]$ & - \\
\hline Heterogeneity: $\mathrm{Tau}^{2}=1.008 ; \mathrm{Chi}^{2}=$ & $=141.50, \mathrm{c}$ & & $P<0.01)$ & $1^{2}=65 \%$ & & 1 \\
\hline & & & & & & 0.4 \\
\hline
\end{tabular}

FIGURE 3. Forest plot of weighted proportions of mortality in studies with sample size for all subgroups. Study identifiers are first-author names and corresponding table entry numbers in Tables 1 and 2 (boldface). Weighted marginal proportions are shown for each subgroup. CBT, Catheter-based studies.

differences in the incidence of CPR in the CBT versus SE groups accounts for the differential mortality between the CBT and SE groups.

\section{DISCUSSION}

We found (Video 1) that published short-term outcomes of both CBT and SE for acute PE are, as expected, of 


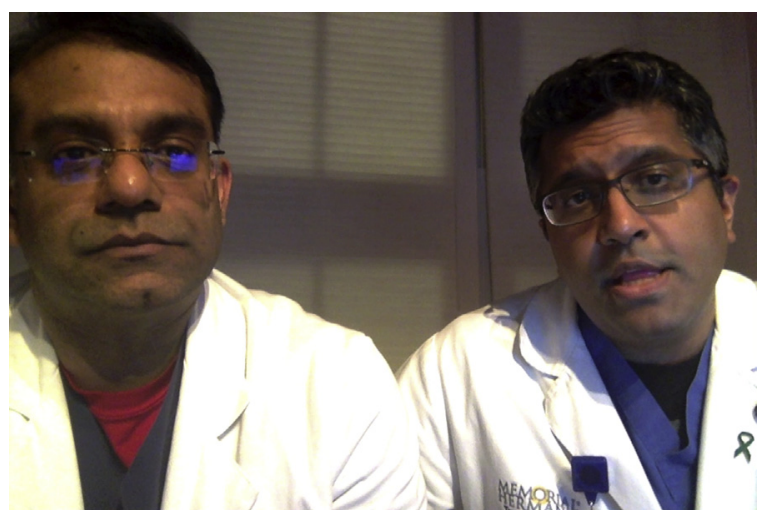

VIDEO 1. Pranav Loyalka, MD, and Keshava Rajagopal, MD, PhD, discuss the findings of their systematic review of catheter-based versus surgical therapies for acute pulmonary embolism. Video available at: https://www.jtcvs.org/article/S0022-5223(18)31520-4/fulltext.

good quality. SE was performed in a more critically ill patient cohort, with a higher proportion of patients with massive PE and a large number of patients with a pretreatment CPR history. In contrast, CBT was undertaken in a less critically ill cohort, with a relatively lower percentage of patients with massive PE. Unadjusted hospital survival outcomes of SE were inferior to CBT, but patients without pre-SE CPR were found to have survival outcomes equivalent to the CBT group as a whole. In addition, CBT was associated with a procedure failure rate of $\sim 8 \%$, which presumably does not exist for SE (although technical success or failure of SE was not documented in the literature surveyed). A majority of CBT studies reported needing to employ an additional modality of CBT distinct from the primary modality to achieve technically or physiologically satisfactory outcomes.

Substantial intercenter variations exist with respect to procedural expertise. As a result, in light of these findings, individual center and practitioner experiences are critically important in determining the best therapeutic strategy. However, some factors should prompt consideration of expanding the role of SE in the management of massive and possibly even submassive PE with high-risk features. First, and perhaps most important, patients with massive PE (and submassive PE on initial presentation that can subsequently evolve massive PE physiology) may require artificial mechanical support of the RV and/or lungs. Venoarterial ECMO and other techniques to mechanically support the RV and/or lungs are most easily undertaken in operating room environments. In centers with ECMO expertise, SE can be undertaken concomitantly or in a delayed fashion ${ }^{70}$ after a period of RV and pulmonary support and end-organ resuscitation. This is perhaps particularly relevant in patients with pretreatment refractory shock and/or CPR. Moreover, in the majority of largevolume medical centers, cardiothoracic surgeons have the greatest experience and facility with these technologies.
CBT, in contrast, rarely is performed by physicians and/or surgeons with experience in advanced mechanical cardiac and/or pulmonary support.

Second, clearance of the embolic burden is perhaps better accomplished via SE. Physiologic end points are of paramount importance, and even in early studies, both SE and CBT have been shown to reduce RV afterload and thereby improve hemodynamic parameters, and to reduce ventilation-perfusion mismatch and thereby improve gas exchange. ${ }^{8,91-93}$ However, some evidence suggests that SE may be more physiologically effective than CBT. For example, in the recently conducted A Prospective, SingleArm, Multi-Center Trial of EkoSonic Endovascular System and Activase for Treatment of Acute Pulmonary Embolism (SEATTLE II) ${ }^{22}$ large, prospective multicenter study of CBT, the mean RV to left ventricle ratio posttreatment was $>0.9$, and mean pulmonary arterial (PA) pressure was reduced from 51.4 to 36.9 ; that is, substantial residual PA hypertension was observed. SE, on the other hand, appears to exhibit better physiologic results, including several of the studies reviewed in our study. Lehnert and colleagues, ${ }^{57}$ in the most recent study we examined, investigated the efficacy of SE versus systemic thrombolysis (albeit not CBT) at removal of the embolic burden in the pulmonary vasculature. Using single-photon emission computed tomography scanning after the procedure, they found that SE was markedly superior to systemic thrombolysis. In addition, Keeling and colleagues $^{94}$ have shown dramatic improvements in ejection phase (ie, inversely proportional to afterload) indices of RV systolic function and tricuspid valve regurgitation.

However, the embolic burden may be nonlinearly related to pulmonary vascular impedance/RV afterload; that is, in critically ill patients with massive PE, small amounts of PE clearance may translate to large reductions in pulmonary vascular impedance and improvements in cardiac output. SE is conducted under direct vision, with the ability to extract a central clot, as well as clots extending into lobar and even segmental branch vessels. In contrast, incompleteness of PE burden clearance from CBT is well recognized, and in fact expected. This is true even of the SEATTLE II study, ${ }^{22}$ which identified only a $30 \%$ improvement in angiographic PE burden, deeming these results procedural success. The mean modified Miller angiographic score based on computed tomography angiography was reduced from 22.5 pretreatment to 15.8 posttreatment. To place this in perspective, a normal score in the absence of any PE is 0 , with a maximum score with complete involvement of all pulmonary vascular zones and totally absent pulmonary blood flow (ie, cardiac arrest) of 40 (the original maximum Miller score based on conventional catheter angiography is 34 ). Particularly in those studies that define procedural success by physiologic 
rather than angiographic criteria, modest PE burden clearance was found to result in physiologic improvements. However, whether further hemodynamic improvements would result from better PE clearance is unclear. Interpretation of surgical data in this regard is complicated. Most patients do not have pre-SE PA catheter data, and PA catheters generally are not placed in the setting of SE. In addition, cardiopulmonary bypass may have adverse effects on pulmonary vascular impedance. ${ }^{95,96}$ Finally, postoperative invasive and even noninvasive positivepressure mechanical ventilation also have variable effects on pulmonary vascular impedance. ${ }^{97,98}$

These arguments in favor of SE notwithstanding (particularly in critically ill patients such as those with massive PE), the optimal treatment of patients with lesser degrees of PE (ie, submassive or less) is unclear. However, a recent multicenter study of SE results, included in our study, may provide insights on this question. Keeling and colleagues $^{60}$ recently reported the results of a multicenter registry of SE. In this group of 214 patients undergoing SE, $82 \%$ had submassive PE, and only $18 \%$ had massive $\mathrm{PE}$; that is, a ratio of massive to submassive $\mathrm{PE}<1$ :4. As noted previously, these demographic characteristics represent not only a much lower risk profile than the existing SE literature, but also are a much lower risk profile than the existing CBT literature. Yet, hospital mortality in the study of Keeling and colleagues ${ }^{60}$ was $11.7 \%$, and in the subgroup of patients who did not experience pre-SE CPR, mortality was still $8.6 \%$. If these findings are reproducible, they would suggest that aggressive use of SE in patients with submassive PE may be unwarranted, due to both apparently inferior survival outcomes relative to CBT, and more importantly, the lack of a survival benefit relative to the natural history of submassive PE.

Our study has several limitations. First, it is retrospective and uncontrolled. Second, when present, studies did not report at what times PE class was determined-at the time of initial presentation to any center, initial presentation to the treating center, or just before initiation of CBT or SE. This is important because submassive PE may deteriorate and convert to massive PE, and deaths due to submassive PE generally are believed to be due to this. Third, etiology of PE generally was not investigated in the studies analyzed. Fourth, mechanisms or modes of death also were not reported in the studies analyzed. Fifth, with respect to SE in particular, technical success or failure was not reported, but rather was presumed. Sixth, long-term follow-up was rare, and studies were limited to in-hospital or $<90$-day outcomes. All of these data, if not absent, may provide important insights into PE and its treatment.

\section{CONCLUSIONS}

Based on the existing data, both SE and CBT have roles in the management of massive and submassive PE.
These results demonstrate that both approaches achieve satisfactory outcomes. SE has practical utility in patients at high risk for needing mechanical RV and/or pulmonary support, and may be superior to CBT in those patients with central PE and larger embolic burden. In contrast, CBT may be better suited to patients for whom cardiac surgery is contraindicated or of extreme risk, and patients with more peripheral (ie, less surgically accessible) disease. Randomized controlled trials, although challenging to conduct in the setting of massive PE with labile patient conditions, will be valuable in identifying appropriate therapies for specific patient subsets.

\section{Conflicts of Interest Statement}

Authors have nothing to disclose with regard to commercial support.

\section{References}

1. Silverstein MD, Heit JA, Mohr DN, Petterson TM, O'Fallon WM, Melton LJ III Trends in the incidence of deep vein thrombosis and pulmonary embolism: a 25-year population-based study. Arch Intern Med. 1998;158:585-93.

2. Castillo C, Tapson VF. Right ventricular responses to massive and submassive pulmonary embolism. Cardiol Clin. 2012;30:233-41.

3. Konstantinides SV. Massive pulmonary embolism: what level of aggression? Semin Respir Crit Care Med. 2008;29:47-55.

4. Tapson VF, Humbert M. Incidence and prevalence of chronic thromboembolic pulmonary hypertension: from acute to chronic pulmonary embolism. Proc Am Thorac Soc. 2006;3:564-7.

5. Di Nisio M, van Es N, Buller HR. Deep vein thrombosis and pulmonary embolism. Lancet. 2016;388:3060-73.

6. Konstantinides SV, Vicaut E, Danays T, Becattini C, Bertoletti L, Beyer-Westendorf $\mathrm{J}$, et al. Impact of thrombolytic therapy on the long-term outcome of intermediate-risk pulmonary embolism. $J \mathrm{Am}$ Coll Cardiol. 2017;69:1536-44.

7. Riera-Mestre A, Jimenez D, Muriel A, Lobo JL, Moores L, Yusen RD, et al Thrombolytic therapy and outcome of patients with an acute symptomatic pulmonary embolism. J Thromb Haemost. 2012;10:751-9.

8. Meyer G, Vicaut E, Danays T, Agnelli G, Becattini C, Beyer-Westendorf J, et al. Fibrinolysis for patients with intermediate-risk pulmonary embolism. $N$ Engl J Med. 2014;370:1402-11.

9. Tibbutt DA, Davies JA, Anderson JA, Fletcher EW, Hamill J, Holt JM, et al. Comparison by controlled clinical trial of streptokinase and heparin in treatment of life-threatening pulmonay embolism. BMJ. 1974;1:343-7.

10. Beall AC Jr, Cooley DA, Debakey ME. Surgical management of pulmonary embolism: experimental and clinical considerations. Dis Chest. 1965;47:382-91.

11. Kilic A, Shah AS, Conte JV, Yuh DD. Nationwide outcomes of surgical embolectomy for acute pulmonary embolism. J Thorac Cardiovasc Surg. 2013;145:373-7.

12. Kuo WT. Endovascular therapy for acute pulmonary embolism. J Vasc Interv Radiol. 2012;23:167-79.

13. Jaff MR, McMurtry MS, Archer SL, Cushman M, Goldenberg N, Goldhaber SZ, et al Management of massive and submassive pulmonary embolism, iliofemoral deep vein thrombosis, and chronic thromboembolic pulmonary hypertension: a scientific statement from the American Heart Association. Circulation. 2011;123:1788-830.

14. Madden LV, Paul PA. Meta-analysis for evidence synthesis in plant pathology: an overview. Phytopathology. 2011;101:16-30.

15. Benedetto U, Melina G, Takkenberg JJ, Roscitano A, Angeloni E, Sinatra R. Surgical management of aortic root disease in Marfan syndrome: a systematic review and meta-analysis. Heart. 2011;97:955-8.

16. Bloomer TL, El-Hayek GE, McDaniel MC, Sandvall BC, Liberman HA Devireddy CM, et al. Safety of catheter-directed thrombolysis for massive and submassive pulmonary embolism: results of a multicenter registry and metaanalysis. Catheter Cardiovasc Interv. 2017;89:754-60.

17. Liang NL, Avgerinos ED, Marone LK, Smith MJ, Singh MJ, Makaroun MS, et al Comparative outcomes of ultrasound-assisted thrombolysis and standard catheter-directed thrombolysis in the treatment of acute pulmonary embolism. Vasc Endovascular Surg. 2016;50:405-10. 
18. Sag S, Nas OF, Kaderli AA, Ozdemir B, Baran I, Erdogan C, et al. Catheterdirected ultrasound-accelerated thrombolysis may be life-saving in patients with massive pulmonary embolism after failed systemic thrombolysis. J Thromb Thrombolysis. 2016;42:322-8.

19. Kabrhel C, Rosovsky R, Channick R, Jaff MR, Weinberg I, Sundt T, et al. A multidisciplinary pulmonary embolism response team: initial 30-month experience with a novel approach to delivery of care to patients with submassive and massive pulmonary embolism. Chest. 2016;150:384-93.

20. Yoo JW, Choi HC, Lee SJ, Cho YJ, Lee JD, Kim HC. Comparison between systemic and catheter thrombolysis in patients with pulmonary embolism. Am J Emerg Med. 2016;34:985-8.

21. Dilektasli AG, Demirdogen Cetinoglu E, Acet NA, Erdogan C, Ursavas A, Ozkaya G, et al. Catheter-directed therapy in acute pulmonary embolism with right ventricular dysfunction: a promising modality to provide early hemodynamic recovery. Med Sci Monit. 2016;22:1265-73.

22. Piazza G, Hohlfelder B, Jaff MR, Ouriel K, Engelhardt TC, Sterling KM, et al. A prospective, single-arm, multicenter trial of ultrasound-facilitated, catheterdirected, low-dose fibrinolysis for acute massive and submassive pulmonary embolism: the SEATTLE II study. JACC Cardiovasc Interv. 2015;8:1382-92.

23. George B, Wallace EL, Charnigo R, Wingerter KE, Kapadia P, Gurley JC, et al. A retrospective analysis of catheter-based thrombolytic therapy for acute submassive and massive pulmonary embolism. Vasc Med. 2015;20:122-30.

24. Kuo WT, Banerjee A, Kim PS, DeMarco FJ Jr, Levy JR, Facchini FR, et al. Pulmonary embolism response to fragmentation, embolectomy, and catheter thrombolysis (PERFECT): initial results from a prospective multicenter registry. Chest. 2015;148:667-73.

25. Engelberger RP, Moschovitis A, Fahrni J, Willenberg T, Baumann F, Diehm N, et al. Fixed low-dose ultrasound-assisted catheter-directed thrombolysis for intermediate and high-risk pulmonary embolism. Eur Heart J. 2015;36:597-604.

26. Dumantepe M, Teymen B, Akturk U, Seren M. Efficacy of rotational thrombectomy on the mortality of patients with massive and submassive pulmonary embolism. J Card Surg. 2015;30:324-32.

27. Quintana D, Salsamendi J, Fourzali R, Narayanan G. Ultrasound-assisted thrombolysis in submassive and massive pulmonary embolism: assessment of lung obstruction before and after catheter-directed therapy. Cardiovasc Intervent Radiol. 2014;37:420-6.

28. Mohan B, Aslam N, Kumar Mehra A, Takkar Chhabra S, Wander P, Tandon R, et al. Impact of catheter fragmentation followed by local intrapulmonary thrombolysis in acute high risk pulmonary embolism as primary therapy. Indian Heart J. 2014;66:294-301.

29. Gaba RC, Gundavaram MS, Parvinian A, Knuttinen MG, Minocha J, Owens CA, et al. Efficacy and safety of flow-directed pulmonary artery catheter thrombolysis for treatment of submassive pulmonary embolism. AJR Am J Roentgenol. 2014; 202:1355-60.

30. Akin H, Al-Jubouri M, Assi Z, Acino R, Sepanski D, Comerota AJ. Catheterdirected thrombolytic intervention is effective for patients with massive and submassive pulmonary embolism. Ann Vasc Surg. 2014;28:1589-94.

31. Kennedy RJ, Kenney HH, Dunfee BL. Thrombus resolution and hemodynamic recovery using ultrasound-accelerated thrombolysis in acute pulmonary embolism. J Vasc Interv Radiol. 2013;24:841-8.

32. Bonvini RF, Roffi M, Bounameaux H, Noble S, Müller H, Keller PF, et al. AngioJet rheolytic thrombectomy in patients presenting with high-risk pulmonary embolism and cardiogenic shock: a feasibility pilot study. EuroIntervention. 2013;8: 1419-27.

33. Nassiri N, Jain A, McPhee D, Mina B, Rosen RJ, Giangola G, et al. Massive and submassive pulmonary embolism: experience with an algorithm for catheterdirected mechanical thrombectomy. Ann Vasc Surg. 2012;26:18-24.

34. Cuculi F, Kobza R, Bergner M, Erne P. Usefulness of aspiration of pulmonary emboli and prolonged local thrombolysis to treat pulmonary embolism. Am J Cardiol. 2012;110:1841-5.

35. Liu S, Shi HB, Gu JP, Yang ZQ, Chen L, Lou WS, et al. Massive pulmonary embolism: treatment with the rotarex thrombectomy system. Cardiovasc Intervent Radiol. 2011;34:106-13.

36. Gao H, Huang GY, Ma LL, Wang LX. Combined catheter thrombus fragmentation and fibrinolysis for acute pulmonary embolism. Intern Med J. 2011;41: 687-91.

37. Ferrigno L, Bloch R, Threlkeld J, Demlow T, Kansal R, Karmy-Jones R. Management of pulmonary embolism with rheolytic thrombectomy. Can Respir J. 2011; 18:e52-8.

38. de Gregorio MA, Laborda A, de Blas I, Medrano J, Mainar A, Oribe M. Endovascular treatment of a haemodynamically unstable massive pulmonary embolism using fibrinolysis and fragmentation. Experience with 111 patients in a single centre. Why don't we follow ACCP recommendations? Arch Bronconeumol. 2011;47:17-24.

39. Yamamoto T, Murai K, Tokita Y, Kato K, Iwasaki YK, Sato N, et al. Thrombolysis with a novel modified tissue-type plasminogen activator, monteplase, combined with catheter-based treatment for major pulmonary embolism. Circ J. 2009; 73:106-10.

40. Lin PH, Annambhotla S, Bechara CF, Athamneh H, Weakley SM, Kobayashi K, et al. Comparison of percutaneous ultrasound-accelerated thrombolysis versus catheter-directed thrombolysis in patients with acute massive pulmonary embolism. Vascular. 2009;17(Suppl 3):S137-47.

41. Chechi T, Vecchio S, Spaziani G, Giuliani G, Giannotti F, Arcangeli C, et al. Rheolytic thrombectomy in patients with massive and submassive acute pulmonary embolism. Catheter Cardiovasc Interv. 2009;73:506-13.

42. Spies C, Khandelwal A, Smith TH, Jolly N, Kavinsky CJ. Percutaneous mechanical thrombectomy for massive pulmonary embolism using a conservative treatment strategy. J Interv Cardiol. 2008;21:566-71.

43. Nakazawa K, Tajima H, Murata S, Kumita SI, Yamamoto T, Tanaka K. Catheter fragmentation of acute massive pulmonary thromboembolism: distal embolisation and pulmonary arterial pressure elevation. Br J Radiol. 2008;81: 848-54.

44. Margheri M, Vittori G, Vecchio S, Chechi T, Falchetti E, Spaziani G, et al. Early and long-term clinical results of AngioJet rheolytic thrombectomy in patients with acute pulmonary embolism. Am J Cardiol. 2008;101:252-8.

45. Kuo WT, van den Bosch MA, Hofmann LV, Louie JD, Kothary N, Sze DY. Catheter-directed embolectomy, fragmentation, and thrombolysis for the treatment of massive pulmonary embolism after failure of systemic thrombolysis. Chest. 2008;134:250-4.

46. Eid-Lidt G, Gaspar J, Sandoval J, de los Santos FD, Pulido T, Gonzalez Pacheco H, et al. Combined clot fragmentation and aspiration in patients with acute pulmonary embolism. Chest. 2008;134:54-60.

47. Pieri S, Agresti P. Hybrid treatment with angiographic catheter in massive pulmonary embolism: mechanical fragmentation and fibrinolysis. Radiol Med. 2007; 112:837-49.

48. Chauhan MS, Kawamura A. Percutaneous rheolytic thrombectomy for large pulmonary embolism: a promising treatment option. Catheter Cardiovasc Interv. 2007;70:121-8.

49. Barbosa MA, Oliveira DC, Barbosa AT, Pavanello R, Kambara A, Egito ES, et al. Treatment of massive pulmonary embolism by percutaneous fragmentation of the thrombus. Arq Bras Cardiol. 2007;88:279-84.

50. Tajima H, Murata S, Kumazaki T, Nakazawa K, Abe Y, Komada Y, et al. Hybrid treatment of acute massive pulmonary thromboembolism: mechanical fragmentation with a modified rotating pigtail catheter, local fibrinolytic therapy, and clot aspiration followed by systemic fibrinolytic therapy. AJR Am J Roentgenol. 2004; 183:589-95.

51. Tajima H, Murata S, Kumazaki T, Nakazawa K, Kawamata H, Fukunaga T, et al. Manual aspiration thrombectomy with a standard PTCA guiding catheter for treatment of acute massive pulmonary thromboembolism. Radiat Med. 2004; 22:168-72.

52. Zeni PT Jr, Blank BG, Peeler DW. Use of rheolytic thrombectomy in treatment of acute massive pulmonary embolism. J Vasc Interv Radiol. 2003;14:1511-5.

53. De Gregorio MA, Gimeno MJ, Mainar A, Herrera M, Tobio R, Alfonso R, et al. Mechanical and enzymatic thrombolysis for massive pulmonary embolism. $J$ Vasc Interv Radiol. 2002;13:163-9.

54. Schmitz-Rode T, Janssens U, Duda SH, Erley CM, Gunther RW. Massive pulmonary embolism: percutaneous emergency treatment by pigtail rotation catheter. $J$ Am Coll Cardiol. 2000;36:375-80.

55. Fava M, Loyola S, Huete I. Massive pulmonary embolism: treatment with the hydrolyser thrombectomy catheter. J Vasc Interv Radiol. 2000;11:1159-64.

56. Schmitz-Rode T, Janssens U, Schild HH, Basche S, Hanrath P, Gunther RW. Fragmentation of massive pulmonary embolism using a pigtail rotation catheter. Chest. 1998;114:1427-36.

57. Lehnert P, Møller CH, Mortensen J, Kjaergaard J, Olsen PS, Carlsen J. Surgical embolectomy compared to thrombolysis in acute pulmonary embolism: morbidity and mortality. Eur J Cardiothorac Surg. 2017;51:354-61.

58. Edelman JJ, Okiwelu N, Anvardeen K, Joshi P, Murphy B, Sanderes LH, et al. Surgical pulmonary embolectomy: experience in a series of 37 consecutive cases. Heart Lung Circ. 2016;25:1240-4.

59. Cho YH, Sung K, Kim WS, Jeong DS, Lee YT, Park PW, et al. Management of acute massive pulmonary embolism: is surgical embolectomy inferior to thrombolysis? Int J Cardiol. 2016;203:579-83. 
60. Keeling WB, Sundt T, Leacche M, Okita Y, Binongo J, Lasajanak Y, et al. Outcomes after surgical pulmonary embolectomy for acute pulmonary embolus: a multi-institutional study. Ann Thorac Surg. 2016;102:1498-502.

61. Neely RC, Byrne JG, Gosev I, Cohn LH, Javed Q, Rawn JD, et al. Surgical embolectomy for acute massive and submassive pulmonary embolism in a series of 115 patients. Ann Thorac Surg. 2015;100:1245-51.

62. Azari A, Beheshti AT, Moravvej Z, Bigdelu L, Salehi M. Surgical embolectomy versus thrombolytic therapy in the management of acute massive pulmonary embolism: short and long-term prognosis. Heart Lung. 2015;44:335-9.

63. Worku B, Gulkarov I, Girardi LN, Salemi A. Pulmonary embolectomy in the treatment of submassive and massive pulmonary embolism. Cardiology. 2014; 129:106-10.

64. Osborne ZJ, Rossi P, Aucar J, Dharamsy S, Cook S, Wheatley B. Surgical pulmonary embolectomy in a community hospital. Am J Surg. 2014;207:337-41.

65. Wu MY, Liu YC, Tseng YH, Chang YS, Lin PJ, Wu TI. Pulmonary embolectomy in high-risk acute pulmonary embolism: the effectiveness of a comprehensive therapeutic algorithm including extracorporeal life support. Resuscitation. 2013;84:1365-70.

66. Aymard T, Kadner A, Widmer A, Basciani R, Tevaerarai H, Weber A, et al. Massive pulmonary embolism: surgical embolectomy versus thrombolytic therapy_-should surgical indications be revisited? Eur J Cardiothorac Surg. 2013; 43:90-4; discussion 94.

67. Taniguchi S, Fukuda W, Fukuda I, Watanabe K, Saito Y, Nakamura M, et al. Outcome of pulmonary embolectomy for acute pulmonary thromboembolism: analysis of 32 patients from a multicentre registry in Japan. Interact Cardiovasc Thorac Surg. 2012;14:64-7.

68. Takahashi H, Okada K, Matsumori M, Kano H, Kitagawa A, Okita Y. Aggressive surgical treatment of acute pulmonary embolism with circulatory collapse. Ann Thorac Surg. 2012;94:785-91.

69. Marshall L, Mundy J, Garrahy P, Christopher S, Wood A, Griffin R, et al. Surgical pulmonary embolectomy: mid-term outcomes. ANZ J Surg. 2012;82:822-6.

70. Malekan R, Saunders PC, Yu CJ, Brown KA, Gass AL, Spielvogel D, et al. Peripheral extracorporeal membrane oxygenation: comprehensive therapy for highrisk massive pulmonary embolism. Ann Thorac Surg. 2012;94:104-8.

71. Zarrabi K, Zolghadrasli A, Ostovan MA, Azimifar A. Short-term results of retrograde pulmonary embolectomy in massive and submassive pulmonary embolism: a single-center study of 30 patients. Eur J Cardiothorac Surg. 2011;40:890-3.

72. Greelish JP, Leacche M, Solenkova NS, Ahmad RM, Byrne JG. Improved midterm outcomes for type A (central) pulmonary emboli treated surgically. $J$ Thorac Cardiovasc Surg. 2011;142:1423-9.

73. Fukuda I, Taniguchi S, Fukui K, Minakawa M, Daitoku K, Suzuki Y. Improved outcome of surgical pulmonary embolectomy by aggressive intervention for critically ill patients. Ann Thorac Surg. 2011;91:728-32.

74. Vohra HA, Whistance RN, Mattam K, Kaarne M, Haw MP, Barlow M, et al. Early and late clinical outcomes of pulmonary embolectomy for acute massive pulmonary embolism. Ann Thorac Surg. 2010;90:1747-52.

75. Sareyyupoglu B, Greason KL, Suri RM, Keegan MT, Dearani JA, Sundt TM III A more aggressive approach to emergency embolectomy for acute pulmonary embolism. Mayo Clin Proc. 2010;85:785-90.

76. Carvalho EM, Macedo FI, Panos AL, Ricci M, Salerno TA. Pulmonary embolectomy: recommendation for early surgical intervention. J Card Surg. 2010;25:261-6.

77. Kukla P, Bryniarski L, Dlugopolski R, Krupa E, Nowak J, Kulak L, et al. Acute pulmonary embolism registry in the Malopolska region - clinical course. Kardiol Pol. 2009;67:735-41.

78. Sadaba JR, Greco E, Alvarez LA, Pulitani I, Juaristi A, Goiti JJ. The surgical option in the management of acute pulmonary embolism. J Card Surg. 2008;23: 729-32.

79. Kadner A, Schmidli J, Schonhoff F, Krahenbuhl E, Immer F, Carrel T, et al. Excellent outcome after surgical treatment of massive pulmonary embolism in critically ill patients. J Thorac Cardiovasc Surg. 2008;136:448-51.

80. Ahmed P, Khan AA, Smith A, Pagala M, Abrol S, Cunninghan JN Jr, et al. Expedient pulmonary embolectomy for acute pulmonary embolism: improved outcomes. Interact Cardiovasc Thorac Surg. 2008;7:591-4.
81. Digonnet A, Moya-Plana A, Aubert S, Fletcher E, Bonnet N, Leprince P, et al Acute pulmonary embolism: a current surgical approach. Interact Cardiovasc Thorac Surg. 2007;6:27-9.

82. Amirghofran AA, Emami Nia A, Javan R. Surgical embolectomy in acute massive pulmonary embolism. Asian Cardiovasc Thorac Ann. 2007;15: 149-53.

83. Spagnolo S, Grasso MA, Tesler UF. Retrograde pulmonary perfusion improves results in pulmonary embolectomy for massive pulmonary embolism. Tex Heart Inst J. 2006;33:473-6.

84. Meneveau N, Seronde MF, Blonde MC, Legalery P, Didier-Petit K, Briand F, et al. Management of unsuccessful thrombolysis in acute massive pulmonary embolism. Chest. 2006;129:1043-50.

85. Sukhija R, Aronow WS, Lee J, Kakar P, McClurg JA, Levy JA, et al. Association of right ventricular dysfunction with in-hospital mortality in patients with acute pulmonary embolism and reduction in mortality in patients with right ventricular dysfunction by pulmonary embolectomy. Am J Cardiol. 2005;95:695-6.

86. Leacche M, Unic D, Goldhaber SZ, Rawn JD, Aranki SF, Couper GS, et al. Modern surgical treatment of massive pulmonary embolism: results in 47 consecutive patients after rapid diagnosis and aggressive surgical approach. J Thorac Cardiovasc Surg. 2005;129:1018-23.

87. Dauphine C, Omari B. Pulmonary embolectomy for acute massive pulmonary embolism. Ann Thorac Surg. 2005;79:1240-4.

88. Yalamanchili K, Fleisher AG, Lehrman SG, Axelrod HI, Lafaro RJ, Sarabu MR et al. Open pulmonary embolectomy for treatment of major pulmonary embolism. Ann Thorac Surg. 2004;77:819-23; discussion 823.

89. Ullmann M, Hemmer W, Hannekum A. The urgent pulmonary embolectomy: mechanical resuscitation in the operating theatre determines the outcome. Thorac Cardiovasc Surg. 1999; 47:5-8.

90. Doerge H, Schoendube FA, Voss M, Seipelt R, Messmer BJ. Surgical therapy of fulminant pulmonary embolism: early and late results. Thorac Cardiovasc Surg. 1999;47:9-13.

91. Greenfield LJ, Reif ME, Guenter CE. Hemodynamic and respiratory responses to transvenous pulmonary embolectomy. J Thorac Cardiovasc Surg. 1971;62: 890-7.

92. Lualdi JC, Goldhaber SZ. Right ventricular dysfunction after acute pulmonary embolism: pathophysiologic factors, detection, and therapeutic implications Am Heart J. 1995;130:1276-82

93. Samoukovic G, Malas T, deVarennes B. The role of pulmonary embolectomy in the treatment of acute pulmonary embolism: a literature review from 1968 to 2008. Interact Cardiovasc Thorac Surg. 2010;11:265-70.

94. Keeling WB, Leshnower BG, Lasajanak Y, Binongo J, Guyton RA, Halkos ME, et al. Midterm benefits of surgical pulmonary embolectomy for acute pulmonary embolus on right ventricular function. J Thorac Cardiovasc Surg. 2016;152: 872-8.

95. Toole JM, Ikonomidis JS, Szeto WY, Zellner JL, Mulcahy J, Deardorff RL, et al Selective endothelin-1 receptor type A inhibition in subjects undergoing cardiac surgery with preexisting left ventricular dysfunction: influence on early postoperative hemodynamics. J Thorac Cardiovasc Surg. 2010;139: 646-54.

96. Viitanen A, Salmenpera M, Heinonen J, Hynynen M. Pulmonary vascular resistance before and after cardiopulmonary bypass. The effect of PaCO2. Chest. 1989:95:773-8.

97. McConnell DH, Maloney JV Jr, Buckberg GD. Postoperative intermittent positive-pressure breathing treatments. Physiological considerations. J Thorac Cardiovasc Surg. 1974;68:944-52.

98. Powers SR Jr, Mannal R, Neclerio M, English M, Marr C, Leather R, et al. Physiologic consequences of positive end-expiratory pressure (PEEP) ventilation. Ann Surg. 1973;178:265-72.

Key Words: pulmonary embolism, surgery, embolectomy, thrombolysis, catheter 